\author{
Zhaoxia Ren · Anren Li • Barker S. Shastry \\ Tirunilai Padma $\cdot$ Radha Ayyagari $\cdot$ Mark H. Scott \\ Marshall M. Parks $\cdot$ Muriel I. Kaiser-Kupfer \\ J. Fielding Hejtmancik
}

\title{
A 5-base insertion in the $\gamma$ C-crystallin gene is associated with autosomal dominant variable zonular pulverulent cataract
}

\author{
Received: 10 January 2000 / Accepted: 14 March 2000 / Published online: 14 April 2000 \\ (C) Springer-Verlag 2000
}

\begin{abstract}
A seven-generation family with 30 members affected by highly variable autosomal dominant zonular pulverulent cataracts has been previously described. We have localized the cataracts to a $19-\mathrm{cM}$ interval on chromosome 2q33-q35 including the $\gamma$-crystallin gene cluster. Maximum lod scores are $4.56(\theta=0.02)$ with D2S157, $3.66(\theta=0.12)$ with D2S72, and $3.57(\theta=0.052)$ with CRYG. Sequencing and allele-specific oligonucleotide analysis of the pseudo $\gamma$ E-crystallin promoter region from individuals in the pedigree suggest that activation of the $\gamma \mathrm{E}$-crystallin pseudo gene is unlikely to cause the cataracts in the family. In addition, base changes in the TATA box but not the Sp1-binding site have been found in unaffected controls and can be excluded as a sole cause of cataracts. In order to investigate the underlying genetic mechanism of cataracts in this family further, exons of the highly expressed $\gamma \mathrm{C}$ - and $\gamma \mathrm{D}$-crystallin genes have been sequenced. The $\gamma \mathrm{D}$-crystallin gene shows no abnormalities, but a 5-bp duplication within exon 2 of the $\gamma \mathrm{C}$-crystallin gene has been found in one allele of each affected family member and is absent from both unaffected family members and unaffected controls. This mutation disrupts the reading frame of the $\gamma \mathrm{C}$-crystallin coding sequence and is pre-
\end{abstract}

Z. Ren · A. Li · M. H. Scott · M. I. Kaiser-Kupfer

J. F. Hejtmancik (®)

National Eye Institute, National Institute of Health,

Bethesda MD 20892, USA

e-mail: f3h@helix.nih.gov,

Tel.: +1 301496 8300, Fax: +1 3014351598

B. S. Shastry

Eye Research Institute, Oakland University,

Rochester, MI 48309-4480, USA

T. Padma

Department of Genetics, Osmania University, Hyderabad, India

R. Ayyagari

Department of Ophthalmology, University of Michigan,

Ann Arbor, Mich., USA

M. M. Parks

Private Practice, Washington D.C. USA dicted to result in the synthesis of an unstable $\gamma \mathrm{C}$-crystallin with 38 amino acids of the first "Greek key" motif followed by 52 random amino acids. This finding suggests that the appropriate association of mutant $\beta \gamma$-crystallins into oligomers is not necessary to cause cataracts and may give us new insights into the genetic mechanism of cataract formation.

\section{Introduction}

Congenital cataracts are common disorders of the eye that often cause blindness in children (Robinson et al. 1987; Foster and Johnson 1990; Lund et al. 1992). Although most commonly inherited in an autosomal dominant manner (Francois 1982; Merin 1991), congenital cataracts are genetically heterogeneous, with clinically identical cataracts mapping to different loci (Hejtmancik 1998). In addition, autosomal dominant congenital cataracts are phenotypically heterogeneous. Although individuals in a family tend to have morphologically similar cataracts (Ionides et al. 1999), identical mutations in the same gene can show intrafamilial phenotypic variability, especially with zonular cataracts. Among the most variable cataracts are the zonular cataracts in the seven-generation family described by Scott et al. (1994).

Crystallins are the major soluble structural proteins in the lens and in humans comprise three major classes, the $\alpha$-, $\beta$ - and $\gamma$-crystallins (Wistow 1990; Ray et al. 1997). The $\beta \gamma$-crystallins have two domains, with each domain being composed of two exceptionally stable protein structures called "Greek key" motifs (Blundell et al. 1981). The $\gamma$-crystallin gene cluster on chromosome 2q33-q35 contains the $\gamma \mathrm{A}$ - (CRYGA), $\gamma \mathrm{B}-(\mathrm{CRYGB}), \gamma \mathrm{C}-(\mathrm{CYRGC})$ and $\gamma \mathrm{D}$ - (CRYGD) crystallin genes, of which $\gamma \mathrm{C}$ - and $\gamma \mathrm{D}$ crystallins are expressed at high levels (Hejtmancik 1998). The $\gamma \mathrm{E}$ - and $\gamma \mathrm{F}$-crystallin pseudogenes are also found in this region. The $\gamma$-crystallins play a crucial role in both lens development and maintenance of lens transparency (Hejtmancik et al. 1995). Several mutations in $\gamma$ crystallin genes have been associated with hereditary 
cataract (Stephan et al. 1999; Heon et al. 1999). Autosomal dominant cataracts in an additional family have been mapped to the region of the $\gamma$-crystallin gene cluster on chromosome 2q33-q35 (Rogaev et al. 1997).

In this report, we map the locus for an autosomal dominant cataract to a region of chromosome 2q33-q35 spanning $19 \mathrm{cM}$ including the $\gamma$-crystallin gene cluster. Although the pseudo $\gamma \mathrm{E}$-crystallin promoter associated with the cataract shows some of the sequence changes described by Brakenhoff et al. (1994), similar changes are also found in several unaffected individuals, suggesting that this gene is unlikely to be the cause of cataracts in this family. Sequencing of the nearby $\gamma \mathrm{D}$-crystallin gene has revealed no changes, but the $\gamma \mathrm{C}$-crystallin gene shows a 5base duplication in exon 2 that is present in all affected family members.

\section{Materials and methods}

\section{Pedigree and patient studies}

These studies were performed under a protocol approved by the NEI Institutional Review Board and conforming to the Declaration of Helsinki. Informed consent was obtained from adult patients and the parents of minor patients. Clinical and ophthalmologic examination included determination of horizontal corneal diameter, intraocular pressure, slit-lamp examination, Snellen visual acuity, stereoacuity, and color vision testing. Detailed medical and family histories were obtained from each available family member. All ophthalmologic examinations were carried out by M.H.S. and M.I.K. Detailed clinical findings of affected individuals were previously described by Scott et al. (1994). A blood sample of $14 \mathrm{ml}$ was obtained from all available members in the family, including affected and unaffected individuals, for transformation into lymphoblastoid lines. Genomic DNA was isolated directly from blood and from transformed lymphocytes as described elsewhere (Smith et al. 1992).

\section{Genotype analyses}

Genotyping was carried out by using microsatellite markers for the polymerase chain reaction (PCR), and products were subsequently separated on $6 \%$ polyacrylamide denaturing gel. PCRs were carried out as described previously (Smith et al. 1992). Briefly, 1.5 nmole of a single primer of each pair was end-labeled with $\alpha^{32} \mathrm{P}$ ATP by incubating for $1 \mathrm{~h}$ at $37^{\circ} \mathrm{C}$ in a reaction mix containing 5 U T4 kinase. After end-labeling, 50 pmole labeled primer was added to the PCR mix containing 50 pmole unlabeled primers (100 pmole for the other pair). Amplification was performed in a thermocycler (Perkin-Elmer 9600) with $60 \mathrm{ng}$ genomic DNA under conditions of denaturing for $30 \mathrm{~s}$ at $94^{\circ} \mathrm{C}$, annealing for $30 \mathrm{~s}$ at $55^{\circ} \mathrm{C}$, and extension for $40 \mathrm{~s}$ at $72^{\circ} \mathrm{C}$ for 30 cycles, followed by final extension for $2 \mathrm{~min}$ at $72^{\circ} \mathrm{C}$. The amplified products were analyzed on the sequencing gel. The gel was dried onto Whatman 3MM filter paper, which was then exposed to Kodak X-Omat film. Each autoradiogram was read independently by three individuals without referring to the disease phenotype. Only results in uniform agreement were used in the analysis. Finally, haplotypes for each individual were constructed (Fig. 1).

\section{Linkage analysis}

Linkage analysis was performed by using the FASTLINK implementation of the LINKAGE program package, version 5.1 (Cottingham et al. 1993; Schaffer et al. 1994). The MLINK program was used for two-point analysis. Allele frequencies were obtained from the Genome Date Base (GDB). Markers in Fig. 1 are shown in the order according to the CEPH/NIH, the CHLC, and the Genethon maps (NIH/CEPH Collaborative Mapping Group 1992; Buetow et al. 1994; Gyapay et al. 1994). Markers D2S157, D2S72, CRYG, D2S104, D2S128, and D2S173 could not be ordered unambiguously by these maps, and their order and distance in centiRays (cR) were determined by screening the Stanford TNG Radiation Hybrid Mapping Panel (Research Genetics, Huntsville, Ala.) and analyzing the results with RHMAP software (Boehnke et al. 1996). Two-point linkage analyses were carried out for the inherited cataracts as a true dominant trait with penetrance of $0.90,0.95$, and 0.98 . Results were obtained assuming $98 \%$ penetrance of the disease.

PCR amplification of $\gamma$-crystallin genes

Human genomic DNA was amplified by PCR with gene-specific primers. The sequences of the primers used for amplification of the $\gamma \mathrm{D}$-crystallin gene (HUMCRYGQ 3-4; Genebank accession nos K03005, K03006) are: Ex-1 (5'CCTTTTGTGCGGTCCTTGCCAACG3' and 5'GCCCAGTCTCTGGCCCCCGCGATGG3'), Ex-2 (5'GGGGGCCAGAGACTGGGGCAGGA3' and 5'CCTGAGGACCTGGGTCCTGAC3'), and Ex-3 (5'CACACTTGCTTTTCTTCTCTTTT3' and 5'TTAAGAAACAACAAGGAGGAC3'). The sequences of the primers used for the amplification of the $\gamma \mathrm{C}$-crystallin gene (HUMCRYGX 3-4; Genebank accession nos. M11970, M11973) are 5' GCATCATCCGTGTCAACCAG 3' and 5'CGGTCGTTACGTCTGATTTATAAA 3'. A sample of $100 \mathrm{ng}$ human genomic DNA was used for PCRs with 3.2 pmole primer in $50 \mu \mathrm{l}$ reaction mix. PCR was carried out in a thermocycler (PerkinElmer 9700). Conditions for amplification of the $\gamma \mathrm{D}$-crystallin gene were a 5 -min denaturing step at $94^{\circ} \mathrm{C}$ followed by 30 cycles, each consisting of $1.5 \mathrm{~min}$ at $94^{\circ} \mathrm{C}, 1 \mathrm{~min}$ at $60^{\circ} \mathrm{C}$, and $2 \mathrm{~min}$ at $72^{\circ} \mathrm{C}$. The conditions for amplification of the $\gamma \mathrm{C}$-crystallin gene were a 5 -min denaturing step at $94^{\circ} \mathrm{C}$ followed by 35 cycles, each consisting of $30 \mathrm{~s}$ at $94^{\circ} \mathrm{C}, 40 \mathrm{~s}$ at $50^{\circ} \mathrm{C}$, and $2 \mathrm{~min}$ at $72^{\circ} \mathrm{C}$.

\section{DNA sequencing}

PCR products were analyzed on $1 \%$ agarose gels and purified by the QIAquick Gel Extraction Kit (QIAGEN, Valencia, Calif.) followed by ethanol/sodium acetate precipitation. Sequencing in the forward and reverse directions with the above exon-specific primers was performed on an ABI 377 prism automated sequencer (ABI, Foster City, Calif.) by using an Amplitaq FS cycle sequencing kit $(\mathrm{ABI})$ with dye-labeled terminators.

Allele-specific oligonucleotide analysis of the pseudo $\gamma \mathrm{E}$-crystallin gene

A 139-bp DNA fragment from the promoter region of intron 1 of the $\gamma$ E-crystallin pseudo gene (Fig. 2) was amplified from the genomic DNA of 30 affected and 16 unaffected individuals by PCR with the following primers: 5'TTGCTGCGGTTCTTGCCAACA3' and 5'CAGGGAGCTACCGGGGGG3' (Brakenhoff et al. 1994). Two allele-specific oligonucleotide (ASO) probes (GGACGATATGTCGGGGCGG, the underlined base having either a $G$ or T substitution) were end-labeled with $\alpha^{32} \mathrm{P}-\mathrm{ATP}$. The PCR-amplified fragments were hybridized separately with the above probes on a slot blot. After being washed $(4 \times 15 \mathrm{~min})$ with Church buffer at room temperature, the blot was then stringently washed at $57^{\circ} \mathrm{C}$ for $5 \mathrm{~min}$ followed by autoradiography. PCR products of both alleles from two affected individuals (14 and 16) and three unaffected individuals $(13,24$, and 92$)$ were also subcloned by TA cloning (Invitrogen, Carlsbad, Calif.), and 8- to 14-allele clones were sequenced for each individual by using the fmol DNA Cycle Sequencing System (Promega, Madison, Wis.) with $\alpha^{33}$ P-ATP-labeled primers (ICN Biomedicals, Costa Mesa, Calif.) to confirm both alleles detected by the ASO assay. 


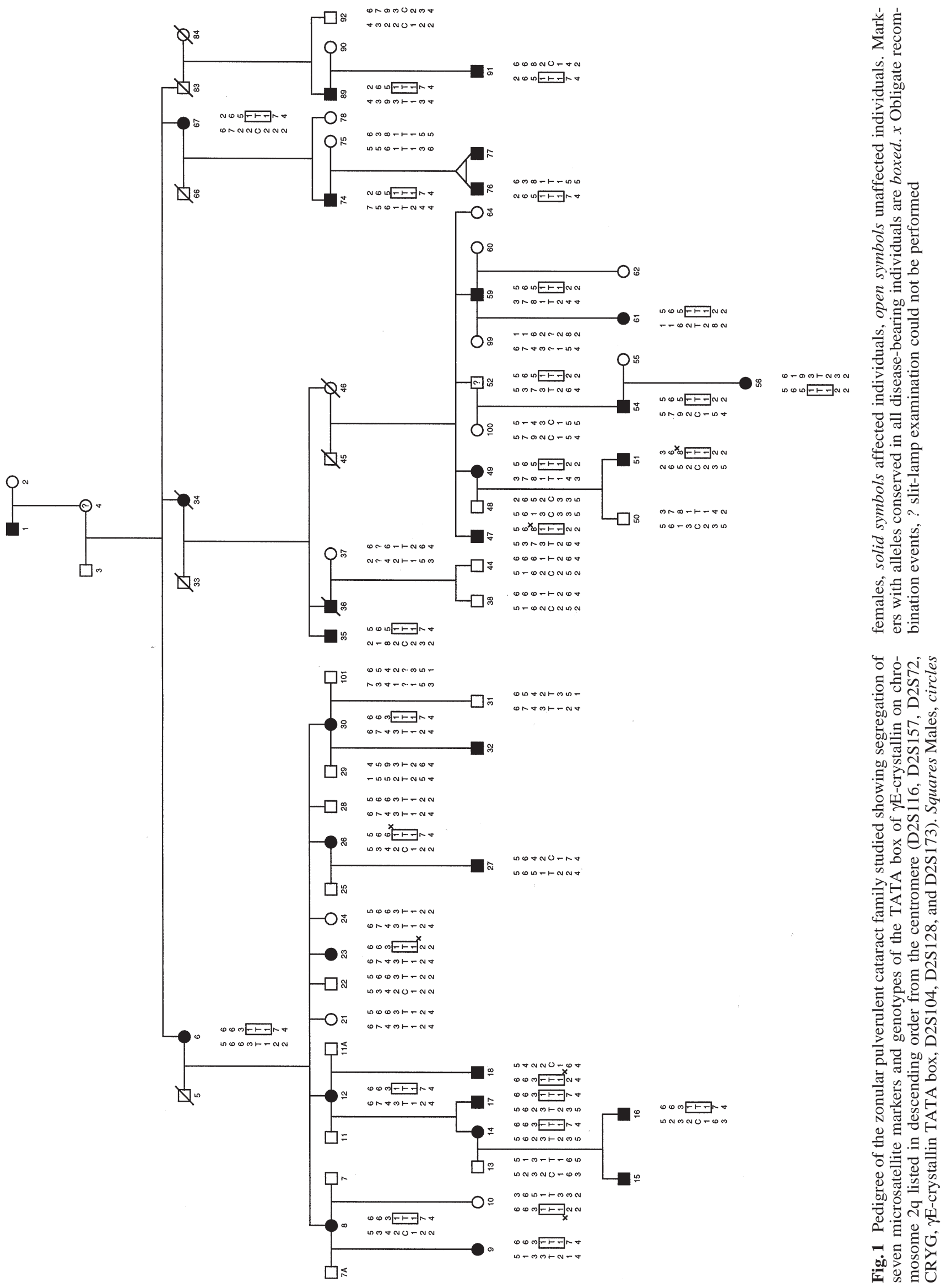


Fig. 2 Comparison of allele sequences of the promoter region of the $\gamma \mathrm{E}$-crystallin gene from the five members of the cataract family with the sequence of the CCL $\gamma \mathrm{E}$-crystallin gene described by Brankenhoff et al. (1994). Identity to the CCL sequence is indicated by dashes and the nucleotide changes are specified. The TATA box is capitalized. Numbers indicating the positions of the sequence correspond to the sequences described in Fig. 3 of Brankenhoff et al. (1994)

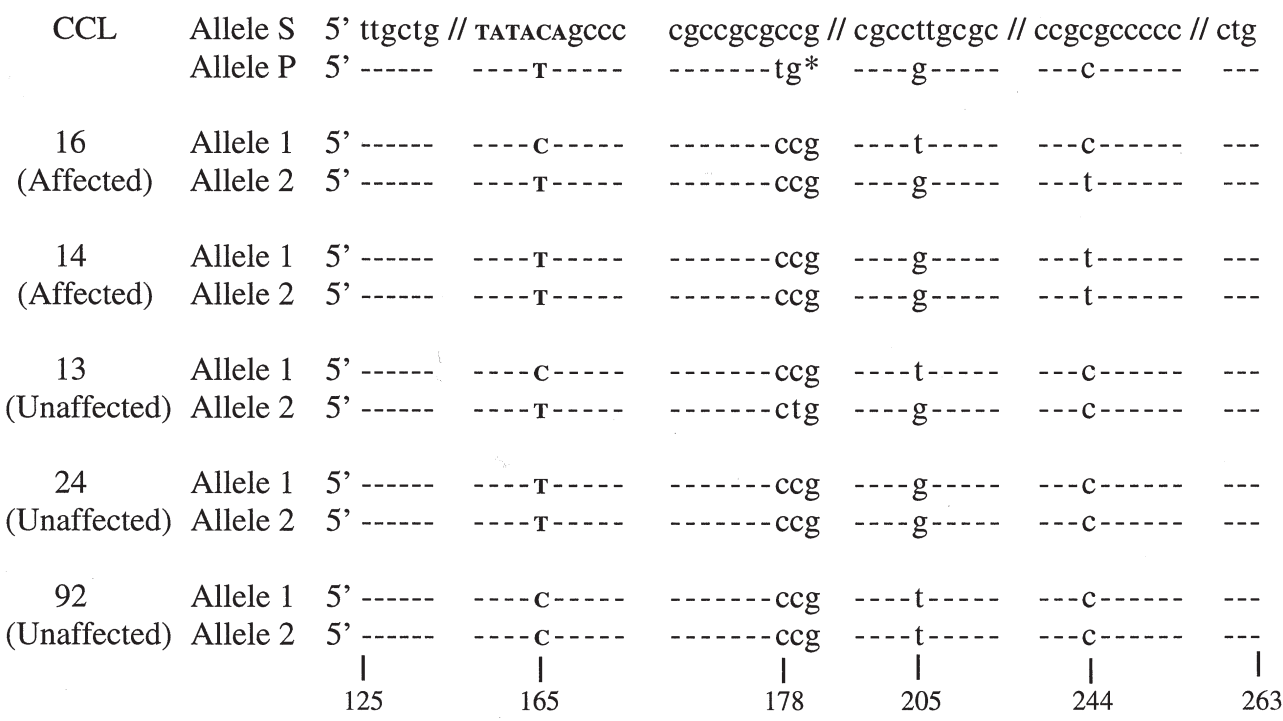

nation, although she had inherited risk alleles for all markers in the critical region from her affected mother, suggesting the possibility of incomplete penetrance (Fig. 1). These studies limited the candidate region to a $19-\mathrm{cM}$ interval between markers D2S116 and D2S173 including the $\gamma$-crystallin gene cluster.

Brakenhoff et al. (1994) have suggested that activation of the $\psi \gamma \mathrm{E}$-crystallin gene resulting from sequence changes of promoter region might be a cause of Coppocklike (CCL) cataracts. To determine whether activation of the $\psi \gamma$ E-crystallin gene was associated with cataracts in this family, the promoter region extending from 36 bases upstream of the TATA box (Fig. 2, base 125) into the first 34 bases of the first intron (Fig. 2, base 263) was sequenced from two affected and three unaffected individuals (Fig. 2). Sequence changes included a $\mathrm{C}$ to $\mathrm{T}$ transition in the TATA box at position 165 , a $\mathrm{T}$ to $\mathrm{G}$ transition at position 205, and a $\mathrm{C}$ to $\mathrm{T}$ transition at position 244 in the first intron. No sequence changes unique to affected individuals were identified. The sequence changes found in the TATA box of affected individuals were analyzed in the remaining family members by ASO analysis of $19 \mathrm{bp}$ of the promoter region of the $\psi \gamma \mathrm{E}$-crystallin, including the TATA box (data not shown). The sequence changes observed following ASO analysis in this region were consistent with changes identified by sequencing analysis, and the sequence changes described above were found in one or even both alleles, not only in affected individuals in this family, but also in 14 unaffected individuals, including ten family members and four unrelated individuals (data not shown). Thus, sequence variations of the $\psi \gamma \mathrm{E}-$ crystallin promoter region are not uniquely associated with the cataracts in this family.

To identify possible mutations in other $\gamma$-crystallin genes in affected family members, exons of the $\gamma \mathrm{C}$ - and $\gamma \mathrm{D}$-crystallin genes were amplified by PCR and sequenced. The $\gamma \mathrm{D}$-crystallin sequence in two affected individuals was identical to that in two normal controls and the published sequence (GenBank accession nos. K03005, combination with the cataract locus. Individual showed a normal phenotype on ophthalmological exami- 


\section{A}

$\underset{220}{\text { GA GA G G GC }} \frac{\text { GC G GC }}{230}$ T GC T G GA T

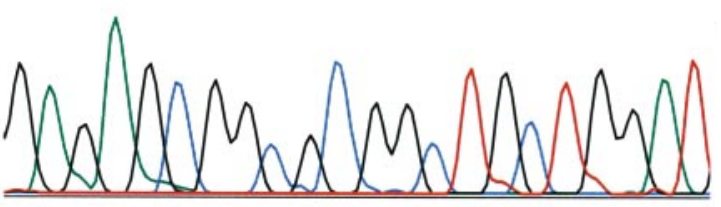

B

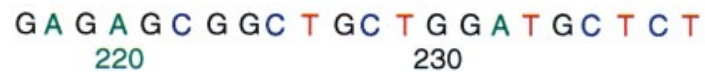

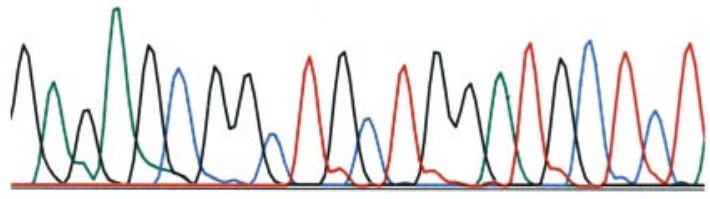

Fig. 3 Sequence analysis of $\gamma \mathrm{C}$-crystallin at exon 2 with the mutation showing a 5-bp repeat (underlined) in one allele (A), and a normal sequence in another allele $(\mathbf{B})$

K03006). A unique 1.7-kb fragment of the $\gamma \mathrm{C}$-crystallin gene was amplified with primers flanking exons $1-3$, and its sequence was determined. Sequences from unaffected individuals 60 and 75 showed complete agreement with the published sequence (den Dunnen et al. 1985), whereas the sequence from affected patients 74 and 76 showed a 5 bp repeat (GCGGC) in exon 2 of the $\gamma \mathrm{C}$-crystallin gene in one allele and the normal sequence in the other (Fig.3). This finding was confirmed by sequencing of subcloned PCR fragments of each allele.

In order to screen for this mutation in the entire family in an efficient and reliable fashion, a 132-bp region including the mutation site was amplified by PCR. With the exception of individual 10, only the 132-bp fragment was amplified in 16 unaffected family members and 46 genetically unrelated unaffected individuals, whereas one allele with 132-bp fragment and a second allele with a 137-bp fragment were amplified in the 30 affected family mem-

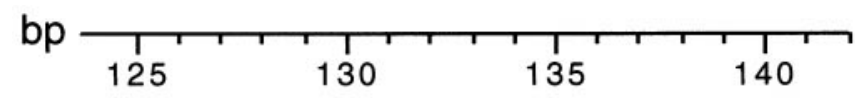

A

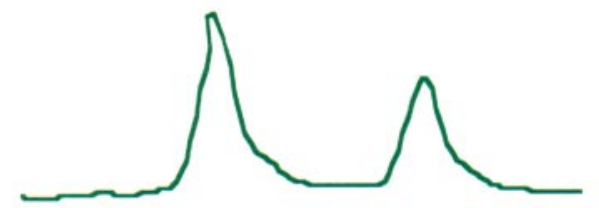

B

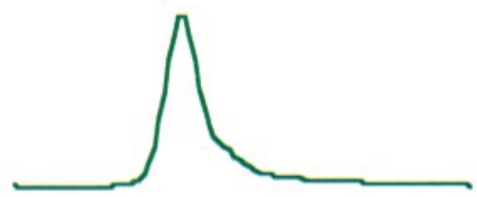

Fig. 4A, B Genotyper analysis of PCR results. Peaks represent fragments from the PCR. A Two fragments (132 bp and $137 \mathrm{bp}$ ) were amplified from affected individual 27 . B Only one fragment (132 bp) was amplified from unaffected individual 17

bers (Fig. 4). Bidirectional direct sequence analysis of the 1.7-kb PCR products from the genomic DNA of two affected family members (12 and 77), two unaffected family members (27 and 62), two unaffected unrelated controls, and individuals 10 and 52 confirmed the above observations. The 5-bp repeat was present only in two affected members of the pedigree, viz., individual 10, who also inherited the risk haplotype in the critical interval from her mother, and individual 52, a clinically unaffected obligate carrier (Fig. 1).

\section{Discussion}

In this report, we localize autosomal dominant congenital cataracts in a seven-generation family to a 19-cM interval on 2q33-q35 between markers D2S116 and D2S173 and show that they are associated with a 5-bp insertion in exon 2 of the $\gamma$ C-crystallin gene.

Brakenhoff et al. (1994) have proposed that the CCL cataract might be caused by over-expression of a truncated $\gamma$-crystallin that is terminated at the end of the first "Greek key" motif and that subsequently produces an improperly folded fragment disrupting the first "Greek key" motif, resulting in an unstable molecule that would cause a lens opacity. Heon et al. (1999) have demonstrated that the cataracts in this family are associated with a single base change causing a T4P amino acid substitution in $\gamma \mathrm{C}$ crystallin and that a similar change in the $\gamma E$-crystallin promoter (Brakenhoff et al. 1994) occurs in unaffected individuals. The present family with autosomal dominant zonular pulverulent cataract also shows sequence changes in the promoter region of $\gamma \mathrm{E}$-crystallin. However, as with the CCL cataract, these changes are observed not only in affected individuals, but also in unaffected and even unrelated individuals (Fig. 2). Similar results have been obtained with both ASO and sequence analyses. These observations show that the sequence changes seen in the $\gamma \mathrm{E}$ - 
crystallin promoter region in this family are not sufficient to cause cataracts by themselves. They also confirm the results of Heon et al. (1999) that the $\mathrm{C}$ to $\mathrm{T}$ transition in the TATA box of the $\psi \gamma \mathrm{E}$-crystallin promoter is unlikely by itself to cause cataracts in the family described by Brakenhoff et al. (1994), although the two-base change in the Sp1-binding region at base 178 could not be addressed directly by either study, as a different sequence change (CCG to CTG vs TGG in the CCL cataract) was found. The CCL cataract is a pulverulent nuclear or nuclear lamellar cataract (Lubsen et al. 1987), which is phenotypically similar to the cataracts in some members of the family described by Scott et al. (1994). However, the autosomal dominant congenital cataracts in this seven-generation family show a much wider range of severity varying from a subtle unilateral zonular pulverulent cataract not impairing visual function to dense bilateral nuclear cataracts resulting in blindness.

Several individuals in this pedigree show minimal or no cataract, in spite of having inherited the haplotypes of the critical interval for this cataract. Among them are individuals 10,12, 14, and 52 (an obligate carrier). All of these individuals also carried 5-base insertions in the $\gamma \mathrm{C}$ crystallin gene. The extreme variation in phenotype from completely asymptomatic individuals with no cataract detectable by portable slit-lamp examination to severe congenital total cataracts as seen in individual 15 (Scott et al. 1994) suggests that, even in these autosomal dominant cataracts, significant effects are exerted by currently uncharacterized factors. The lack of a significant correlation between phenotypic severity in parent-children pairs (Scott et al. 1994) argues against a major effect by modifying genes. In addition, the occurrence of individuals with a severe cataract in one eye and no detectable cataract in the other suggests a random variation in the phenotype, since the effects of modifying genes and environment should be similar for both eyes. Scott et al. (1994) have raised the possibility that this radical phenotypic variability might result from a requirement for somatic mutation of the second $\gamma \mathrm{C}$-crystallin gene in the developing lens for cataracts to occur. However, whereas it remains a formal possibility, this hypothesis is difficult to test.

In addition to the association of the CCL cataract with a T4P mutation in $\gamma \mathrm{C}$-crystallin and a fetal nuclear needlelike aculeiform cataract with an $\mathrm{R} 58 \mathrm{H}$ mutation in $\gamma \mathrm{D}$ crystallin (Heon et al. 1999), two mutations in $\gamma \mathrm{D}$-crystallin associated with congenital cataracts have also been reported recently. These include a dominant punctate cataract first appearing in the first year of life and progressing to total cataract in childhood associated with an R14C mutation on the surface of $\gamma \mathrm{D}$-crystallin (Stephan et al. 1999), and an interesting congenital cataract consisting of crystallized $\gamma \mathrm{D}$-crystallin within lens fiber cells resulting from an R37S mutation in this molecule (Kmoch et al. 1999). These suggest that cataracts can result from mutations that do not affect the stability and tertiary structure of $\gamma$-crystallins but that cause surface changes that affect their association.
We have demonstrated that a novel mutation with a 5bp duplication within exon 2 of the $\gamma \mathrm{C}$-crystallin gene is present in all affected individuals including one obligate carrier (individual 52, Fig. 1). Whereas the insertion of small direct repeats has been associated with slipped mispairing during gene replication (Cooper and Krawczak 1991), there are no obvious repeats or inversions in the immediate vicinity of this insertion. Unlike the single base change (AAGATCACCTTCTAT to AAGATCCCCTTCTAT at nucleotide 411 in the mRNA encoding KITFY to KIPFY) recently described in $\gamma \mathrm{C}$-crystallin (Heon et al. 1999), the 5-bp repeat disrupts the reading frame of the $\gamma \mathrm{C}$-crystallin coding sequence and results in synthesis of a truncated polypeptide consisting of 38 amino acids from the first "Greek key" motif followed by 52 random amino acids. Because the two motifs forming the amino-terminal domain have interdependent hydrogen bonding (Blundell et al. 1981; Slingsby and Clout 1999), this truncated polypeptide would be predicted not to have the ability to fold properly to form the first "Greek key" motif. Neither would it have the interdomain receptor through which $\beta \gamma$-crystallin domains interact (Sergeev and Hejtmancik 1997). Even single amino acid changes in this area can prevent interdomain binding and destabilize not only the mutant, but also the interacting domain (Palme et al. 1997). Thus, it should not be able to associate in a normal swapped-domain fashion with other $\beta$ - or $\gamma$-crystallins. Presumably, an unstable monomeric protein is formed that eventually leads to lens opacity and causes the cataract. Therefore, these findings suggest that normal association of mutant and normal $\beta \gamma$-crystallins through the hydrophobic interdomain interfaces into oligomers is not necessary to cause cataracts.

It is unclear why this seemingly drastic $\gamma \mathrm{C}$-crystallin mutation causes minimal or no cataracts in some lenses and congenital total cataracts in others, whereas the T4P substitution results in a more consistent nuclear lamellar cataract. The physiological effect of this mutation on the properties of $\gamma$-crystallin remains to be elucidated. Currently, we are making a transgenic mouse model of this human cataract; such a model should provide a valuable tool for the study of strategies for human cataract prevention and gene therapy.

Acknowledgements We are grateful to family members for their participation. We also thank J. Redman for her expert assistance in collecting information for control samples.

\section{References}

Blundell T, Lindley P, Miller L, Moss D, Slingsby C, Tickle I, Turnell B, Wistow G (1981) The molecular structure and stability of the eye lens: X-ray analysis of gamma-crystallin II. Nature 289:771-777

Boehnke M, Lunetta K, Hauser E, Lange K, Uro J, Vandertoep J (1996) RHMAP: statistical package for multipoint radiation, 3.0 http:www.hgmp.mrc.ac.uk./Registered/Option/rhmap.html

Brakenhoff RH, Henskens HAM, Rossum MWPC van, Lubsen NH, Schoenmakers JGG (1994) Activation of the gammaEcrystallin pseudogene in the human hereditary Coppock-like cataract. Hum Mol Genet 3:279-283 
Buetow KH, Weber JL, Ludwigsen S, Scherpbier-Heddema T, Duyk GM, Sheffield VC, Wang Z, Murray JC (1994) Integrated human genome-wide maps constructed using the CEPH reference panel. Nat Genet 6:391-393

Cooper DN, Krawczak M (1991) Mechanisms of insertional mutagenesis in human genes causing genetic disease. Hum Genet 87:409-415

Cottingham RW, Idury RM, Schaffer AA (1993) Faster sequential genetic linkage computations. Am J Hum Genet 53:252-263

Dunnen JT den, Moormann RJ, Cremers FPM, Schoenmakers JGG (1985) Two human gamma-crystallin genes are linked and riddled with Alu-repeats. Gene 38:197-204

Foster A, Johnson GJ (1990) Magnitude and causes of blindness in the developing world. Int Ophthalmol 14:135-140

Francois J (1982) Genetics of cataract. Ophthalmologica 184:6171

Gyapay G, Morissette J, Vignal A, Dib C, Fizames C, Millasseau P, Marc S, Bernardi G, Lathrop M, Weissenbach J (1994) The 1993-1994 Genethon human genetic linkage map. Nat Genet 7:246-339

Hejtmancik JF (1998) The genetics of cataract: our vision becomes clearer. Am J Hum Genet 62:520-525

Hejtmancik JF, Kaiser-Kupfer MI, Piatigorsky J (1995) Molecular biology and inherited disorders of the eye lens. In: Scriver CR, Beaudet AL, Sly WS, Valle D (eds) The metabolic basis of inherited disease, vol 7. McGraw Hill, New York, pp 4325-4349

Heon E, Priston M, Schorderet DF, Billingsley GD, Girard PO, Lubsen N, Munier FL (1999) The gamma-crystallins and human cataracts: a puzzle made clearer. Am J Hum Genet 65: $1261-1267$

Ionides A, Francis P, Berry V, Mackay D, Bhattacharya S, Shiels A, Moore A (1999) Clinical and genetic heterogeneity in autosomal dominant cataract. Br J Ophthalmol 83:802-808

Kmoch S, Asfaw B, Bezouska K, Brynda J, Sedlacek J, Filipec M, Elleder M (1999) Cataract due to crystal deposits of $37 \mathrm{R} \rightarrow \mathrm{S}$ mutated gammaD-crystallin. Am J Hum Genet 65:A305

Lubsen NH, Renwick JH, Tsui LC, Breitman ML, Schoenmakers JG (1987) A locus for a human hereditary cataract is closely linked to the gamma-crystallin gene family. Proc Natl Acad Sci USA 84:489-492

Lund AM, Eiberg H, Rosenberg T, Warburg M (1992) Autosomal dominant congenital cataract; linkage relations; clinical and genetic heterogeneity. Clin Genet 41:65-69

Merin S (1991) Inherited cataracts. In: Merin S (ed) Inherited eye diseases. Dekker, New York, pp 86-120
NIH/CEPH Collaborative Mapping Group (1992) A comprehensive genetic linkage map of the human genome. Science 258: $67-86$

Palme S, Slingsby C, Jaenicke R (1997) Mutational analysis of hydrophobic domain interactions in gamma B-crystallin from bovine eye lens. Protein Sci 6:1529-1536

Ray ME, Wistow G, Su YA, Meltzer PS, Trent JM (1997) A1M1, a novel non-lens member of the beta-gamma-crystallin superfamily associated with the control of tumorigenicity in human malignant melanoma. Proc Natl Acad Sci USA 94:3229-3234

Robinson GC, Jan JE, Kinnis C (1987) Congenital ocular blindness in children, 1945 to 1984 . Am J Dis Child 141:1321-1324

Rogaev EI, Rogaeva EA, Korovaitseva GI, Farrar LA, Petrin AN, Keryanov SA, Turaeva S, Chumakov I, St.George-Hyslop P, Ginter EK (1997) Linkage of polymorphic congenital cataract to the gamma-crystallin gene locus on human chromosome 2q33-35. Hum Mol Genet 5:699-703

Schaffer AA, Gupta SK, Shriram K, Cottingham RW (1994) Avoiding recomputation in genetic linkage analysis. Hum Hered 44:225-237

Scott MH, Hejtmancik JF, Wozencraft LA, Reuter LM, Parks MM, Kaiser-Kupfer MI (1994) Autosomal dominant congenital cataract: interocular phenotypic heterogeneity. Ophthalmology 101:866-871

Sergeev YV, Hejtmancik JF (1997) A method for determining domain binding sites in proteins with swapped domains: implications for betaA3- and betaB2-crystallins. In: Marshak DR (ed) Techniques in protein chemistry, vol 8. Academic Press, New York, pp 817-826

Slingsby C, Clout NJ (1999) Structure of the crystallins. Eye 13:395-402

Smith RJH, Lee EC, Kimberling WJ, Daiger SP, Pelias MZ, Keats BJB, Jay M, Bird A, Reardon W, Guest M, Ayyagari R, Hejtmancik JF (1992) Localization of two genes for Usher syndrome type I to chromosome 11. Genomics 14:995-1002

Stephan DA, Gillanders E, Vanderveen D, Freas-Lutz D, Wistow G, Baxevanis AD, Robbins CM, VanAuken A, Quesenberry MI, Bailey-Wilson J, Juo SH, Trent JM, Smith L, Brownstein MJ (1999) Progressive juvenile-onset punctate cataracts caused by mutation of the gammaD-crystallin gene. Proc Natl Acad Sci USA 96:1008-1012

Wistow G (1990) Evolution of a protein superfamily: relationships between vertebrate lens crystallins and microorganism dormancy proteins. J Mol Evol 30:140-145 